\title{
Development Status of Gas Diffusion Coefficient in China
}

\author{
Haifeng Chen1, Yuechao Teng1, Shuangfang Lu2 \\ 1 Northeast Petroleum University The formation mechanism of oil and gas reservoir and resource evaluation \\ Heilongjiang Province key laboratory, Daqing, Heilongjiang, China, 163318 \\ 2 China University of Petroleum(Hua Dong), Qingdao, Shandong, China, 266001
}

\begin{abstract}
As the world's major oil exploration and development is deepening, the importance of gas exploration and development is increasing. As an important constant of evaluating gas saving and loss, diffusion coefficient of gas cap is of great significance in the theoretical study and practical application for natural gas exploration and development. Based on a lot of researching and summarizing of literatures, this paper analyzes the main controlling factors, theoretical and practical application of calculation methods of gas diffusion coefficient, and summarizes the current status and focuses on academic research, proposing some problems, then made several prospects.
\end{abstract}

Key words: - Gas cap Controlling factor Diffusion coefficient Closed feature

\section{INTRODUCTION}

Diffusion is a process that substance effected by concentration gradient spontaneously moves from high concentration to low concentration area, the power of diffusion is the concentration differences of substance in space ${ }^{[1]}$. Diffusion coefficient is a physical describes diffusion rate of gas in the formation, reflects the gas diffusion strength of formation. Gas diffusion coefficient also has many applications in actual production and research. such as cap closed evaluation, estimating loss amount and reserve amount of gas.So accurately obtain diffusion coefficient has always been a key factor restricting the study of gas preservation and loss.Many scholars had done numerous exploratory theoretical and experimental work in the past, And has made a series of impressive results ${ }^{[1]-[11]}$.

\section{CONTROLLING FACTORS AND QUANTITATIVE CALCULATION METHODS OF DIFFUSION COEFFICIENT}

\section{A. Diffusion of gas}

Diffusion of gas is influenced by many factors, but academics mostly describe the diffusion of natural gas by using Fick's law. Under stationary non-homogeneous movement condition, Gas diffusion obeys Fick's first law:

$$
\frac{d m}{d t}=-D A \frac{d c}{d x}
$$

In the formula above: $\frac{d m}{d t}$ is the amount of gas diffusion of per unit time, $(\mathrm{mg} / \mathrm{s}) ; \mathrm{D}$ is the diffusion coefficient, $\left(\mathrm{cm}^{2} / \mathrm{s}\right) ;$ A is cross-sectional area of the gas diffusion flow passes, $\left(\mathrm{cm}^{2}\right) ; \frac{d c}{d x}$ is concentration gradient; $\mathrm{C}$ is molecular concentration;The negative sign indicates the direction of gas diffusion flow is from high concentration to low concentration. 


\section{B. The main controlling factors of diffusion coefficient}

As a physical charactering the gas diffusion strength in the formation, diffusion coefficient mainly controls by several factors below ${ }^{[2]}$ :

\section{a.Types of gas}

As natural gas is diffused through the pore of cap,different types of hydrocarbon molecules have different diameters. Therefore, the smaller the molecular diameter of hydrocarbons, the stronger diffusion capacity, more easily pass the pores of the cap, the greater diffusion coefficient.

\section{b.Properties of gas cap rock}

The porosity and the curvature of the pore and other factors can also affect gas diffusion coefficient. According to derived linear regression equations and fitted curves based on measurement data in the laboratory by scholars[3] can be known: The larger the porosity and the median diameter of the cap rock, the larger the diffusion coefficient; the larger the bending of pores, the lesser diffusion coefficient.

\section{c. Formation temperature}

Diffusion movement is in the state of molecules in essence. Temperature is an important factor affecting the rate of molecular motion, the two is a positive correlation. To gas, is easy to be known, The higher the formation temperature, the greater the diffusion coefficient; otherwise smaller diffusion coefficient.

\section{d. Properties of pore fluid}

Cap rock pore fluid occupied pore space of cap rock, can hinder the diffusion of gas. The larger water saturation of the cap, the smaller diffusion coefficient. In addition, The higher the formation water salinity, the smaller solubility and the amount of gas molecules forms a hydrated gas, also makes a lower diffusion rate gas passes through the formation water, and then makes a smaller diffusion coefficient.

\section{Quantitative calculation of diffusion coefficient}

Over the years, a number of domestic and foreign researchers proposed some equations measuring diffusion coefficient. The more common are the following three equations:

a.Former Soviet scholar Antonov (1954) summarized empirical formula based on the relation between cap diffusion coefficient and total porosity[4]:

$$
D=A \varphi \times 10^{-8}
$$

In the formula above: D is the diffusion coefficient $\left(\mathrm{cm}^{2} / \mathrm{s}\right)$; A is a petrophysical constant associated with regional constant.

\section{b.Corrected Einstein's equation}

$$
D=\frac{K T}{6 \pi \gamma \mu} \bullet \frac{\Phi}{\tau}
$$

In the formula above: $\mathrm{K}$ is Boltzman constant, $1.38 \times 10^{-23} \mathrm{~J} / \mathrm{K}^{\text {; }}$ is temperature $(\mathrm{K})$; $\Phi$ is porosity; $\gamma$ is radius of gas molecules $(\mathrm{m}) ; \mu$ is the viscosity of formation water $(\mathrm{Pa} \cdot \mathrm{s}) ; \tau$ is the bending rate of formation pore;

c.Nesterov (1971) proposed an equation by regression analysis based on measuring a large amount of diffusion coefficient data: 


$$
D=(K \times 15 \varphi) \times 10^{-8} \times \operatorname{alg} 1.2 \varphi \times 10^{-8}
$$

In the formula above: $\varphi$ is shale total porosity; $\mathrm{K}$ is a coefficient describes mudstone pore and particle size;

There are also researchers proposed some regression formula based on experimental data ${ }^{[3]}$, and got excellent application effects.

\section{RESEARCH STATUS OF DIFFUSION COEFFICIENT}

From the experimental point of view, gas diffusion coefficient is not directly measured by the experimental devices, but firstly measures the amount of gas diffusion passes rock samples within a certain time $^{[5]}$,then calculates out diffusion coefficient according to Fick's law. But the passing time of gas through the rock samples is too long, and is too difficult to test; And under the effect by high temperature for a long time, water in the rock samples can easily evaporate, thus greatly influence the test results ${ }^{[6]}$. So now common method is to measure the diffusion coefficient of dry rock samples in the laboratory then regulates. Generally first make core to a certain diameter cylindrical samples, measuring after discharging pore water and gas within the rock sample $^{[7]}$.

Foreign experimental determination for the diffusion coefficient has an earlier start, now the most developed experimental method is proposed by Krooss.B Et al in $1987^{[8]}$. However, this method can only be measured at atmospheric pressure, and can not apply radial confining pressure on the rock samples, experimental temperature is comparatively lower, can not accurately simulate the actual geological conditions.

Chinese scholars also proposed some experimental determination methods for diffusion coefficient after many years of study. Hao Shisheng proposed a technology that measures diffusion coefficient of rock by using airtight diffusion chamber in $1989^{[5]}$ by establishing a closed diffusion chamber at both ends of the core to save the high-pressure gas, sampling in the two chambers in the beginning and end of the experiment. This method takes the actual geological conditions of the rock under high temperature and pressure condition into account, and applies confining pressure. But this method take a manual sampling method, and has only two data points, Therefore, lacks of precision experimental results.

Some scholars have proposed new methods after, as a diffusion coefficient measuring device in 1995 designed by Wan Yu Jin, which used a gas chromatograph ${ }^{[9]}$, overcomes the shortage of manual sampling methods, and has a simple structure, easy to operate. However, when the diffusion chamber at both ends of water-saturated rock samples is evacuated will cause a great water loss in rock samples and make measurement unfavorable. Also in 1994 the oil industry publishing house "gas migration and accumulation balancing its application" and 1997 Hohai University Press published "migration and accumulation of oil and gas accumulation model" proposed their experimental determination of diffusion coefficient technologies. But there is a serious loss of water in water-saturated rock samples when evacuating at both ends of the diffusion chamber. In 2004 Chen Weijun et al, who devised an experimental device to prevent the loss of water in water-saturated rock samples ${ }^{[10]}$.

Diffusion movement is caused by the effect of concentration gradient, so it may adversely affect the gas diffusion of the experimental determination in the process of adding pressure on rock samples. For this case, LI Zhisheng et al disclosed an diffusion coefficient measurement experimental technique which is not affected by the pressure in $2012^{[11]}$.

China's experimental method for measuring the diffusion coefficient has got a later start, but studied by above scholars has reached the world advanced level. 
In theory, research work of diffusion coefficient started earlier abroad, theories of molecular diffusion had been initially formed in the late nineteenth century. In middle of last century, some Soviet scholars applied the theories to the aspects of gas reservoirs and achieved many significant results ${ }^{[4]}$. In the seventies and eighties of last century, the basic theoretical research on gas diffusion coefficient had been basically perfect.

Former Soviet scholar Antonov summarized the relationship between the diffusion coefficient and porosity of cap cover in $1954^{[3]}$,but this relationship failed to fully take factors such as formation temperature, formation water and other fluid properties that affects the diffusion coefficient into account. In 1971 Nesterov proposed a more complete regression formula to calculate the diffusion coefficient on the basis of regression analysis based on measuring numerous diffusion coefficient data, the regression equation takes pore shape factor into account.

Currently, the methods for diffusion coefficient calculation of domestic scholars and foreign scholars method are broadly consistent, both firstly summarize the regression formula based on the actual situation of the work area, then conducting regulation taking various factors into account ${ }^{[2][4][6][7]}$. The most common regulation formula is Corrected Einstein's equation, This formula can conduct diffusion coefficient regulation taking into account of viscosity and temperature of formation water.

The cap rock is made up with mudstone and sandstone; these two have different diffusion coefficients. Obviously, the diffusion coefficient of the cap rock is related to proportions of mudstone and sandstone. Through the establishment of cap rock volume model ${ }^{[12]}$, by using gas diffusion coefficient of mudstone and sandstone and there thickness ratio, consolidating gas diffusion coefficient of the cap rock by series principle.

\section{CONCLUSION AND PROSPECTS}

Due to the complexity of the geological conditions of the cover, although there are many ways to obtain diffusion coefficient, however, there are limitations of each method. Now diffusion coefficient obtaining still exists some room for development, some theoretical and experimental methods is still to be improved. We should take on a global perspective, fully consider the impact of various factors; using more flexible means and applies them in combination. To propose models and formulas more in line with the actual situation, thereby more accurate obtaining diffusion coefficient.

In this paper, combining the current status of research of diffusion coefficient, for the future direction of development are the following prospects:

A.Currently on rock samples generally take biaxial compression pressure method in the laboratory. Triaxial compression method can be taken in the future in order to simulate actual rock samples in underground geological conditions better.

B.After a long period of geological evolution, the constant such as physical properties and temperature which affect diffusion coefficient must be constantly changing ${ }^{[13][14]}$. In order to more accurately estimate the amount of gas loss, future research should identify methods that more precisely restore the ancient gas diffusion coefficient.

C.There is a big difference between the geological conditions of the cap in the underground and which in laboratory, resulting in changes of the amount of parameters. The current regulation process generally only considers the influence of pore fluid and temperature. Taking other parameters into consideration of regulation, may be more accurately obtain the diffusion coefficient.

\section{REFERENCES}

[1] Huang Zhilong. Quantitative evaluation and application of concentration closed of the gas cap [J]. Acta Sedimentologica Sinica, 1996, 14(2): 89-94. 
[2] Zhang Jing. Methods of rock diffusion coefficient [J]. Henan Petroleum, 1996, 10(6): 11-15.

[3] Li Jingkun. Method of calculating the amount of gas diffusion [J]. Xinjiang Petroleum Geology, 1999, 20(5): 383-386.

[4] Antonov P L. On the diffusion permeability of some claystones[J].Sb Geokhi Met Poisk Nefti I Gaza, 1954,2:39-55.

[5] Hao Shisheng. Study of diffusion coefficient of light hydrocarbon and gas migration, accumulation, balancing principle [J]. Acta Petrolei Sinica, 1991, 12(3): 17-24.

[6] Fu Guang. Measured gas diffusion coefficient correction at formation conditions [J].Advances in Earth Sciences, 2001, 16(4): 484-489

[7] Li Haiyan. Study of Experiment of gas diffusion coefficient [J]. Petroleum Geology \& Experiment, 2001, 23(1): 108-112.

[8] Kross B M, Leythaeuser D,Schaefer R G. The quantification of diffusive hydrocarbon losses through cap rocks of natural gas reservoir — a reevaluation. AAPG Bulletin,1992,76(3),403-406.

[9] Chinese Petroleum Corporation Oil \& Gas Exploration and Development Research Institute, Langfang Branch. A measuring device for the diffusion coefficient of rock: China, CN94224552.0[P].1995-08-02.

[10] China Petroleum \& Chemical Corporation.Diffusion coefficient tester: China, CN200410090629.6 [P].2004-11-10.

[11] China National Petroleum Corp. measurement equipment and determination method for diffusion coefficients of hydrocarbons in rock under high temperature and pressure: China, CN201210465895.7[P]. 2012-11-16.

[12] Fu Guang.Methods and applications of gas diffusion coefficient in inhomogeneous cap rock [J]. Daqing Petroleum Geology and Development, 2004, 23(3): 1-3.

[13] Hao Jingbo.Ancient gas diffusion coefficient recovery method for sand shale formation and its application [J].Daqing Petroleum Geology and Development, 2007, 26(6): 16-19.

[14] Fu Guang. Recovery methods of ancient gas diffusion coefficient [C]. Beijing: International Symposium on hydrocarbon accumulation mechanism and oil and gas resource assessment, 1996. 